\title{
Experimental assessment of multiple contact wear using airborne noise under dry and lubricated conditions
}

\author{
M A Khan ${ }^{1 *}$, Kanza Basit ${ }^{1}$, S Z Khan ${ }^{1,2}$, K A Khan ${ }^{3}$, A G Starr ${ }^{1}$ \\ ${ }^{1}$ School of Aerospace, Transport and Manufacturing, Cranfield University, \\ Cranfield, United Kingdom \\ 2 Department of Mechanical Engineering, Islamic University of Madinah, \\ Madinah, Saudi Arabia \\ ${ }^{3}$ Department of Aerospace Engineering, Khalifa Universtiy of Science, Technology and \\ Research, Abu Dhabi, United Arab Emirates \\ *Corresponding Author: muhammad.a.khan@cranfield.ac.uk
}

\begin{abstract}
:
Generation of wear and airborne noise is inevitable in mechanical contacts of machine components. This paper addresses the effectiveness of airborne noise data in estimating the wear on a disc under multi-contact conditions. A pin on disc rig was employed to study the role of noise parameters on the evolution of the wear area. When a pin slides on the disc, the airborne noise is generated and subsequently a sound signal is obtained. These signals, for various sets of experiments, were recorded using a digital microphone. A Matlab code was developed and employed to estimate noise parameters from the recorded sound. Noise parameters including values of voltage RMS, noise counts and amplitudes of dominant frequencies were used to analyze the variation in the disc wear at different time intervals. These parameters were found to be effective in the determination of wear damage evaluation under different loads without lubrication.
\end{abstract}

\section{Keywords:}

Wear, airborne noise, diagnosis, multi contact, lubrication

The final, definitive version of this article has been published in the Journal,Proceedings of the Institution of Mechanical Engineers, Part J: Journal of Engineering Tribology, First published March 29, 2017, (C <SAGE Publications Ltd, Journals Online: http://online.sagepub.com/doi/10.1177/1350650117700341 


\section{Introduction}

Airborne noise and wear phenomena are inevitable in machine components even working in normal operational conditions. Surface degradation, at the mechanical contacts due to wear, has an impact on the noise emitted because of the rapid release of energy from localized sources within a material [1]. Severity of surface degradation can markedly change the spectrum of noise, and hence an analysis of emitted noise can lead to the possible health diagnosis of machine components [2 - 4].

Noise signals have been used to study friction and wear phenomena since the late 1970's [5].The effectiveness of these signals has been tested mostly on rotating machines and manufacturing tool wear [6-14], while their trend spectra are often analyzed to determine the relationship between the noise and the wear [12, 16-20].Tang et al observed trend based spectra and performed experiments on a gear rig. They concluded that the wear can cause significant changes in the amplitudes of the harmonics of gear meshing frequency [12]. Hisakado and Warashina measured the emitted noise count rates, the extent of damage on worn surfaces, specific wear rates and the coefficient of friction on single point of mechanical contact [16]. They provided a relationship between the surface damage and the count rates without considering lubrication. Bereznyakov and Stadnichenko modeled a relationship between the friction and the alteration of the surface energy [17]. They found that the noise emission is directly proportional to the sliding velocity and the wear intensity. Similar research provided a relationship between the acoustic emissions, the dissipation energy, the contact load and the sliding speed [19-21]. Noise signals have also been studied under the effect of lubrication [22-24]. Recently, researchers have shown the possibility of the trend modeling between noise signals and the wear in terms of accumulated 
signal energy and changes in spectrum amplitude [10, 24-27]. However, analysis of airborne noise signals for the wear evaluation of the mechanical components with multiple contact points under load and a lubricated test scheme is still not available and requires an in-depth study.

In this work, experiments were designed and performed to identify the effectiveness of the airborne noise analysis for the evaluation of wear in a mechanical component with multiple contacts. A series of experiments was performed on a pin on disc rig under different loads with and without lubrication. Multiple stainless steel (SS) pins were mounted on a rotating mild steel (MS) disc to generate wear and emitted noise to resemble real machine noise (i.e. wear on multiple contact points at the same time). Other pin materials' were used to observe the wear phenomena. During analysis, signal parameters including root mean square (RMS) values, counts and cumulative amplitudes of dominated frequencies (ADF), along with microscopic images of the worn out surfaces, were used to identify the efficacy of the airborne noise for wear diagnosis.

\section{Methodology}

A pin on disc sliding wear test rig was used for the experimentation as shown in Figure 1 (a) and (b). The setup was composed of four metallic arms to act as pin holders which were assembled at $0^{\circ}, 90^{\circ}, 180^{\circ}$ and $270^{\circ}$ on a metal frame. A circular mild steel disc was fastened to a shaft which was connected to a motor with a v-belt. The motor was operated at constant speed of $250 \mathrm{rpm}$, which was checked by an rpm meter. The pins contacted with the disc at a radius of $50 \mathrm{~mm}$ from the disc center. The surface of the disc was initially turned on a lathe and ground with a 400 grit sand paper. These two processes were used to prepare a uniform contact surface before the start of each experiment. Stainless steel pins were used with a diameter of $5 \mathrm{~mm}$ and contact diameter of $0.04 \mathrm{~mm}$. One end of the pins was sharpened to have a tip angle of $120^{\circ}$ which formed its 
shape like a cone; hence its tip acted as a point contact on the disc and increases the amount of wear. The other end of the pins had threads up to $10 \mathrm{~mm}$ in length. These threads were used to mount the weights and for loading the contacts as shown in Figure 1(b). Due to the design of the pin holders, a load of $4.9 \mathrm{~N}$ was exerted on the contacts in all the experiments and considered as normal load condition. An oil pump was used to lubricate the contact points with the drip lubrication near the contact point at a rate of $5 \mathrm{ml} / \mathrm{sec}$ on each pin. Motor oil of $10 \mathrm{~W}-30$ grade with a density of $877 \mathrm{~kg} / \mathrm{m}^{3}$ was used as a lubricant. Fresh oil was used for each interval of all the experiments.

In order to record the noise generated by the contacts, a non contact technique is adapted. A microphone was vertically placed at the center of the disc and equally distanced to each pin by using a slot in the transparent acrylic box as shown in Figure 1(a). The microphone can measure frequencies of up to $8 \mathrm{kHz}$ similar in range as used in some recent research $[27,28]$. It is connected to a computer for recording noise for further analysis. The sampling frequency during recording was set at $44.1 \mathrm{kHz}$ with 16 bit sampling. Images of the wear damage annulus on the disc were taken from 6 different positions with a portable microscope (AM413T Dino-Lite Pro) at a magnification of 100x and 200x as shown in Figure 2. The use of microscopic images to measure effective wear diameter is an average approximation to calculate the worn out area of the disc. Such a method has previously been used for wear debris and acoustic emission analysis $[14,16]$. By referring to the ASTM Standards, the wear volume can also be calculated with the measured disc worn out diameter, considering no or very less pin wear [29]. But it was found that the area and the volume both exhibit a similar trend under mentioned conditions. Therefore, in this research, worn out area is used to express the material wear as it has been used as a degree of damage since 1990s [30]. 
A total of eight experiments were performed under different loads and lubrication conditions. For brevity these are summarized in Table 1 . All the experiments were repeated three times, while their average was considered during the analysis.

Table 1: List of Experiments

\begin{tabular}{|c|c|c|c|}
\hline Experiment no. & Contact points & Lubrication & Load \\
\hline 1 & \multirow[t]{4}{*}{$\begin{array}{l}2 \text { pins }(\text { Same } \\
\text { materials, }\end{array}$} & No & Normal \\
\hline 2 & & No & Yes $(4.91 \mathrm{~N})$ \\
\hline 3 & & Yes & Yes $(4.91 \mathrm{~N})$ \\
\hline 4 & & No & Yes $(9.81 \mathrm{~N})$ \\
\hline 5 & \multirow[t]{3}{*}{$\begin{array}{l}4 \text { pins } \quad \text { (Same } \\
\text { materials, }\end{array}$} & No & Normal \\
\hline 6 & & No & Yes $(4.91 \mathrm{~N})$ \\
\hline 7 & & Yes & Yes $(4.91 \mathrm{~N})$ \\
\hline 8 & $\begin{array}{l}2 \text { pins (Different pin } \\
\text { materials, One CS and } \\
\text { the other SS) }\end{array}$ & No & Normal \\
\hline
\end{tabular}

The duration of each experiment was 6 minutes. Noise parameters of the recorded signals were analyzed in 36 equal segments of time, each with an interval of 10 seconds. In parallel, the variation in the disc wear area was calculated after every 120 seconds by measuring the wear damage from the captured images. This led us to correlate the signal parameters with the wear evolution.

The raw noise signals recorded by the microphone were in voltage sine waves. Any change in noise can produce effects on the recorded waves. The high sampling frequency made it difficult to observe these effects on all the possible sampling points. This actually led us to calculate the parameter of RMS by taking the root mean square of the noise signal. This can not only converge the recorded sample points but also provide an immediate response if there is any change in raw noise. The noise counts were calculated in Matlab $\subset$ on the basis of rule base algorithm by setting a threshold voltage value of 0.095 volts. Signal amplitude values above this threshold were 
counted and added to represent the noise counts. The threshold value was obtained by running the rig in a close room condition without any pin on disc contact/s. Values lower than the threshold was predominantly generated by the motor and hence noise counts parameter filtered all the background noise. The cumulative amplitude of dominant frequencies was obtained by applying fast Fourier transformation on the raw noise signal. Theoretically, the asperities of pin and disc are responsible to generate noise in a pin on disc setup. In each rotation of the disc, the sliding action of the disc asperities against the pin asperities can cause noise with distinct frequencies. It can be assumed that any change in asperitical behavior can represent the material wear and eventually affects the amplitude of frequency response of the generated noise. Therefore, the values of this amplitude can also be used for analyzing the behavior during material wear. In all the experiments, four distinct peaks were observed on frequency spectra, whose amplitudes were showing trends with the recorded noise values. The amplitudes of these peaks were cumulatively added and assumed as the third noise parameter. The cumulative amplitude was further analyzed with the worn out area at different time intervals.

\section{Results and discussion}

The noise signals were recorded in the time domain for all the experiments. These signals were processed in a Matlab $@$ code, which was purposely develop to obtain signals parameters for every 10 seconds during the experiments. For example, the recorded time domain signal and the calculated parameters for Experiment 1 are shown in Figures 3 and 4 respectively.

The values of noise signal parameters were further normalized for comparative analysis. The means of these normalized trends were also calculated for three 120 seconds time intervals and hence made them comparable with the variation in the disc wear area. For an example, calculation of the variation in the disc wear area for Experiment 1 is described here. 
The microscopic images of wear damages with their respective time intervals for Experiment 1 are shown in Figure 5. After the first interval, the disc image showed a wear damage width of $0.386 \mathrm{~mm}$ i.e. the difference of the outer and inner radii's as illustrated in the Figure 6.

As the disc damage area form an annulus, the effective disc wear area can be calculated by Equation 1:

Wear damage area (1st Interval, 1 st experiment $)=\pi\left(r_{o}{ }^{2}-r_{i}{ }^{2}\right)=121.280 \mathrm{~mm}^{2}$

Similarly, effective disc wear areas were calculated as $267.035 \mathrm{~mm}^{2}$ and $426.087 \mathrm{~mm}^{2}$ for interval 2 and interval 3 respectively. The variation in the disc wear area was further calculated by subtracting the effective wear area of any interval with its preceding interval. In Experiment 1, it was calculated for all the three intervals as: $121.280 \mathrm{~mm}^{2}, 145.755 \mathrm{~mm}^{2}$ and $159.052 \mathrm{~mm}^{2}$. The same approach was applied on the images of the disc wear captured during all the experiments as shown in Figure 7.

In a no-load with no lubrication condition (Experiment 1), the noise signal parameters and wear evolution are shown in Figure 8. It can be observed that with the increasing parameters, the variation in the wear area is also increased. It is important to note that in this experiment the maximum wear area was found at the third interval (between 240-360 seconds).

For a $4.9 \mathrm{~N}$ load with no lubrication (Experiment 2), the maximum wear was observed in the first interval (0-120 seconds). The behaviour of the corresponding noise signal parameters in this experiment is shown in Figure 9. 
When a 4.9 $\mathrm{N}$ load with lubrication (Experiment 3) was applied over 2 SS pins, the maximum values of noise parameters were found in the last interval (240-360 seconds), but the maximum wear on the disc was observed in the second interval as shown in Figure 10. In a $9.81 \mathrm{~N}$ load condition with no lubrication (Experiment 4), the first interval had the maximum wear area and the maximum values of recorded signals parameters as shown in Figure 11. The possible reason for this deviation could be the additional noise generated when a lubricant is added due to contact of pin to lubricant particles before contact to disc [21].

These measurements showed a clear relationship between the variation in disc wear and the maximum mean values of noise signal parameters. In all the 2 pin experiments, the maximum values of parameters and the variation in the wear area were obtained in the same time interval, except when the lubrication was used. The reason for this contrast was the varied nature of lubrication mechanics and its tribological effects at the contact points. The magnitudes of the compared parameters were observed without a specific trend for the same time intervals under different operational (i.e. loading and lubrication) conditions.

Similarly, results were obtained for 4 point contacts under the operational conditions as mentioned in Table 1. For a no load and no lubrication condition (Experiment 5), the maximum values were found to be in the third interval (240-360 seconds) as shown in Figure 12. In $4.90 \mathrm{~N}$ load and no lubrication condition (Experiment 6), maximum values were found at the first interval (0-120 seconds) as shown in Figure 13. Similarly to the 2 pin Experiment 3, in $4.91 \mathrm{~N}$ load and lubrication condition (Experiment 7), the values of maximum wear area and noise signal parameters were not found in the same interval as shown Figure 14. By comparing the above results, it can be seen that the SS pins showed the same behavior for similar dry conditions (non lubricated) irrespective of the number of pins used and the load applied. For a dry direct 
contact without any external load, noise and wear parameters increase linearly as confirmed from the literature $[13,21]$. When an external load was added, the two parameters are found maximum. In the start of the experiments, an increase in number of asperitical contacts causing airborne noise and consequently surface wear occurs rapidly. While under lubrication, these parameters did not lie in the same interval. The reason was the presence of lubricant between the pin and disc which generated an additional noise [21].

An experiment was also performed using a carbon steel pin and a stainless steel pin over the mild steel disc. The same methodology was adapted and it was found that even if the contact material changes, it does not affect the relationship between the noise signal parameters and the wear area. In this experiment, like stainless steel pins, the maximum values of the parameters and the maximum worn out areas both were observed in the same interval i.e. 120-240 seconds as shown Figure 15.

\section{Conclusion}

A multi-point contact phenomenon was studied experimentally. Airborne noise parameters were used to analyze the effectiveness of the wear diagnosis and its quantification. The following conclusions were derived from the results and discussion.

I. Values of noise signals parameters can be used directly to estimate the wear area and its variation. 
II. Under dry conditions, the maximum wear in multi-point contacts can be identified by the maximum values of the noise parameters. This is independent of the number and the material of contact points and the applied load.

III. Under lubricated conditions, conclusion II is not valid. We found that the varied nature of lubrication mechanics and tribological effects might be a dominant factor at the contact locations. However, further experiments need to be performed to confirm this hypothesis, which is beyond the scope of the present study.

It has been well established in the literature that noise signals parameters can directly used to identify wear. But with the set of experiments performed in this study, we conclude that a discrete or functional quantification between the evolution of wear area and noise signals parameters is also possible. To obtain this nature of quantification, large number of experiments must be performed under dry and wet conditions to cater variations in operational conditions.

\section{References}


1. Xiaoli Li. A brief review: acoustic emission method for tool wear monitoring during turning. International Journal of Machine Tools \& Manufacture 2002; 42: 157-165.

2. Alan H, Masaki W, Hiroshi M. The relationship between acoustic emissions and wear particles for repeated dry rubbing. Wear 2008; 265: 831-839.

3. Shirakashi T, Gong W, Obikawa T. In-process monitoring of tool damage by active method — behavior of damping ratio with tool wear development. Seimitsu Kogaku Kaishi/Journal of the Japan Society for Precision Engineering 1995; 61: 1750-1754.

4. Dybala J, Zimroz R. Rolling bearing diagnosing method based on Empirical Mode Decomposition of machine vibration signal. Applied Acoustics 2014; 77: 195-203.

5. Gandhi O P, Sharma J P. Signature response for friction and wear. ASLE Transactions $1979 ; 22: 365-368$.

6. Anon. Vibration and wear detection in rotating machinery by acoustics analysis. Applied Acoustics 1989; 28: 213-219.

7. Sadat A, Shivakumar R. Detection of tool flank wear using acoustic signature analysis. Wear 1987; 115: 265-272.

8. Silva R H L, Silva M B, Hassui A. A probabilistic neural network applied in monitoring tool wear in the end milling operation via acoustic emission and cutting power signals. Machining Science and Technology 2016; 20: 386-405.

9. Prakash M, Kanthababu M, Rajurkar K P. Int j Adv Manuf Technol 2015; 77:1499-1511.

10. Trabelsi H, Kannatey-Asibu E. Tool wear and sound radiation in metal cutting. ASME Prod. Engg. Div. 1990; 45: 121-131.

11. Duspara M, Sabo K, AntunStoić. Acoustic emission as tool wear monitoring. Tehničkivjesnik 2014; 21: 1097-1101 
12. He T, Yuanhui W, Huaiqiao Y, Zhang Ce. Quantitative diagnosis of gear wear by spectrum analysis of noise. Journal of Tainjin University 1987; 2: 22-30.

13. Kagnaya T, Boher C, Lambert L et all. Wear mechanisms of WC-Co cutting tools from high speed tribological tests. Wear 2009; 267: 890-897.

14. Hase A, Wada M, Koga T et all. The relationship between acoustic emission signals and cutting phenomenon in turning process. Int. J. Adv. Manuf. Technol. 2014; 70: 947-955.

15. Chiou R Y, Liang S Y. Analysis of acoustic emission in chatter vibration with tool wear effect in turning. Int. J. of Mach. Tools and Manuf. 2000; 40: 927-941.

16. Hisakado, Warashina. Relationship between friction and wear properties and acoustic emission characteristics: iron pin on hardened bearing steel disk. Wear 1998; 216: 1-7.

17. Bereznyakov, Stadnichenko. Correlation of acoustic emission characteristics from friction unit surface and tribological characteristics. Friction and Wear 2014; 19: 312317.

18. Yan W, O’Dowd N P, Busso E P. Numerical study of sliding wear caused by a loaded pin on a rotating disk. J. of the Mech. and Phys. of Sol. 2002; 50: 449-470.

19. Ito S, Shima M, Jibiki T et all. The relationship between AE and dissipation energy for fretting wear. Tribol. Int. 2009;42: 236-242.

20. Fan Y, Gu F, Ball A. Modelling acoustic emissions generated by sliding friction. Wear 2010; 268: 811-815.

21. Benabdallah H S, Aguilar D A. Acoustic emission and its relationship with friction and wear for sliding contact. Tribol. Trans.2008; 51: 738-747.

22. Edmonds J, Resner M S, Shkarlet K. Detection of precursor wear debris in lubrication systems. In: Aerospace Conference, NJ, USA, $25^{\text {th }}$ March 2000, paper no. 6805502, pp 73-77. New York: IEEE. 
23. Miettinen Jand Andersson P. Acoustic Emission of rolling bearings lubricated with contaminated grease. Tribol. Int. 2000; 33: 777-787.

24. Filonenko S, Kosmach A. Acoustic emission in the friction of composite materials. Aviation 2010; 18: 57-63.

25. Ahmaida A, Dong Z, Gu F, Ball A. Gear wear process monitoring using acoustic signals. In: 21st International Congress on Sound and Vibration (ICSV), Beijing, China, $13-17$ July 2014. AL, USA: International Institute of Acoustics and Vibrations.

26. Hase A, Wada M, Mishina H. Microscopic study on the relationship between AE signal and wear amount. Wear 2013; 308: 142-147.

27. Karim Z, Nuawi M Z, Ghani J A et all. Sliding wear evaluation of aluminum alloy (7075T6) on hardened steel (AISI4340) via non-contact technique by multi-level analysis. Wear 2015; 334-335: 99-104.

28. Ghazali M J, Mansor N I I, Nuawi M Z, Ghani J A. Facile wear failure monitoring of commercial bearing alloys using I-kaz method. Engg. Fail. Anal. 2013; 35: 545-553

29. ASTM Committee G02 on Wear and Erosion. G99-95a: Standard Test Method for Wear Testing with a Pin-on-disk Apparatus: ASTM; 2000

30. Jiaa C L, Dornfield D. Experimental studies of sliding friction and wear via acoustic. Wear 1990; 139: 99-104. 
2017-03-29

\section{Experimental assessment of multiple contact wear using airborne noise under dry and lubricated conditions}

Khan, Muhammad

SAGE Publications (UK and US)

Khan MA, Basit K, Khan SZ, et al., (2017) Experimental assessment of multiple contact wear using airborne noise under dry and lubricated conditions, Proceedings of the Institution of Mechanical Engineers, Part J: Journal of Engineering Tribology, Volume 321, Issue 12, December 2017, pp. 1503-1516

http://dx.doi.org/10.1177/1350650117700341

Downloaded from Cranfield Library Services E-Repository 